\title{
Comparison of Electron Tomographic Reconstructions from Different Types of Signals
}

\author{
H. Friedrich*, M.R. McCartney**, and P.R. Buseck*** \\ * Department of Geological Sciences, Arizona State University, Tempe, AZ, 85287 \\ ** Center for Solid State Science, Arizona State University, Tempe, AZ, 85287 \\ *** Department of Geological Sciences and Department of Chemistry/Biochemistry, \\ Arizona State University, Tempe, AZ, 85287
}

During the past decade an increasing number of materials whose function is controlled by their microstructure have been studied using transmission electron microscopy (TEM). Since TEM is a projection technique, the 3D information in a single micrograph is limited. One solution is electron tomography, which reconstructs the 3D structure using a stack of 2D projections at different tilt angles (tilt-series). The theory of $3 \mathrm{D}$ reconstruction requires a signal proportional to the projected property, which excludes signals affected by diffraction contrast. We are applying electron tomography to crystalline samples and wish to determine the effects of diffraction contrast on the 3D reconstruction.

Magnetite crystals produced by magnetotactic bacteria strain MV1 were investigated using a Tecnai F20. Automated acquisition of bright field (BF) TEM and annular dark field (ADF) STEM tilt-series (150 and $300 \mathrm{~mm}$ camera lengths) were performed with Emispec ${ }^{\circledR}$ Tomography component for the Tecnai Imaging and Analysis software package. Each tilt-series contains 141 images ranging from -70 to +70 degrees tilt at 1 degree increments. The $3 \mathrm{D}$ structure was reconstructed running IDL® scripts on a Windows 2000 platform. The scripts based on the simultaneous iterative reconstruction technique (SIRT) [1] were programmed and implemented in IDL $®$ by Matthew Weyland [2]. The results were further analyzed using Digital Micrograph image processing software.

The reconstruction results shown in FIG 1., FIG. 2. and FIG. 3. are similar. Focus changes during acquisition result in visible Fresnel fringes in the BF reconstruction and blurring in the ADF reconstructions. Since only a small number of the 141 images contain strong diffraction contrast the averaging effect of the reconstruction distributes this additional contrast over the whole reconstruction volume. By using different signals (BF, ADF at different camera lengths) we varied the amount of diffraction contrast contributing to the reconstruction to study the effect on the apparent densities of the reconstructed crystals. To estimate the apparent densities, we calculated the sum of the intensities $\left(\mathrm{N}_{\mathrm{A}}, \mathrm{N}_{\mathrm{B}}\right)$ along paths $\mathrm{A}$ and $\mathrm{B}$ through the centers of the crystals divided by path lengths A and B (indicated on FIG. 1.,2. and 3.). These densities should be the same if diffraction contrast has no influence on the reconstruction. In the case of ADF STEM (150 mm camera length) the ratio of the densities (smaller grain to larger grain) was close to 1, while the ratio was 1.1 for BF TEM and 1.2 for ADF STEM (300 mm camera length). This trend is consistent with an increasing contribution of diffraction contrast to image formation, which results in a higher apparent density of smaller crystals [3].

\section{References}

[1] P. Gilbert, J. Theor. Biol. 36 (1972) 105 
[2] M. Weyland, PhD thesis, Cambridge (2001)

[3] Funding is provided by NASA through the ASU Astrobiology center, grant number NCC2-1051.

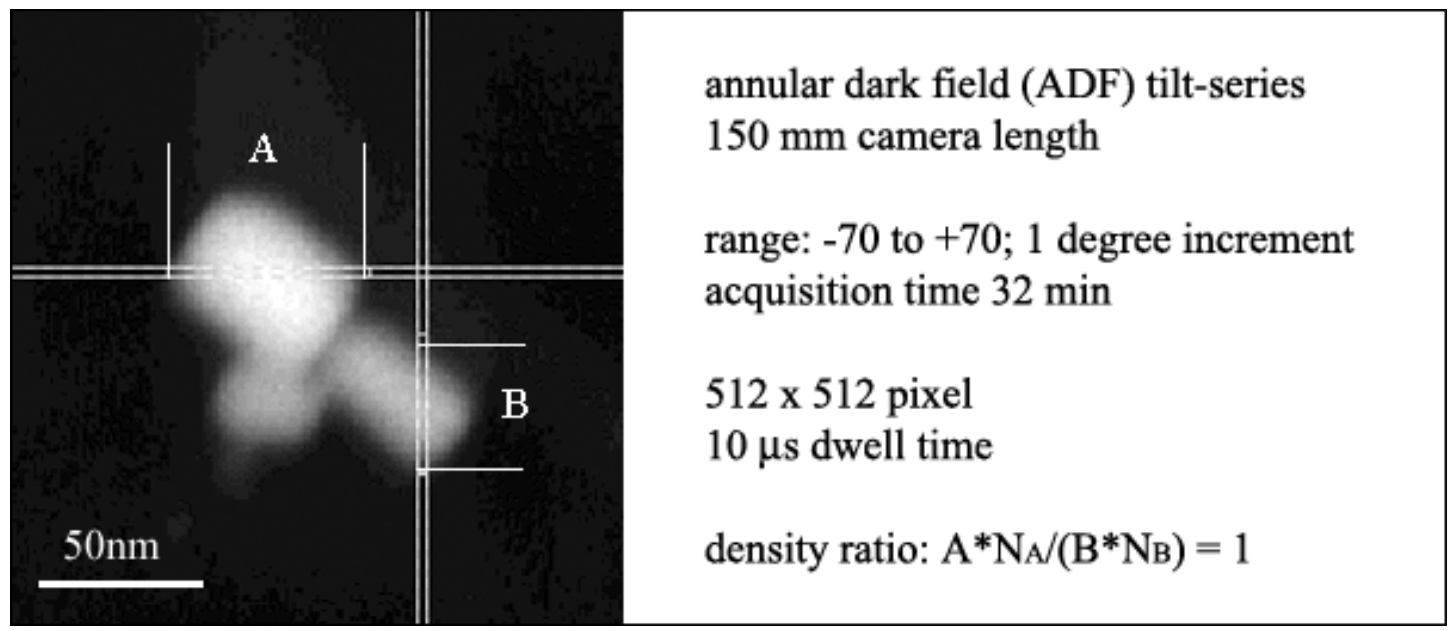

FIG. 1 Projection along $\mathrm{z}$ axis of reconstructed volume from annular dark-field tilt-series

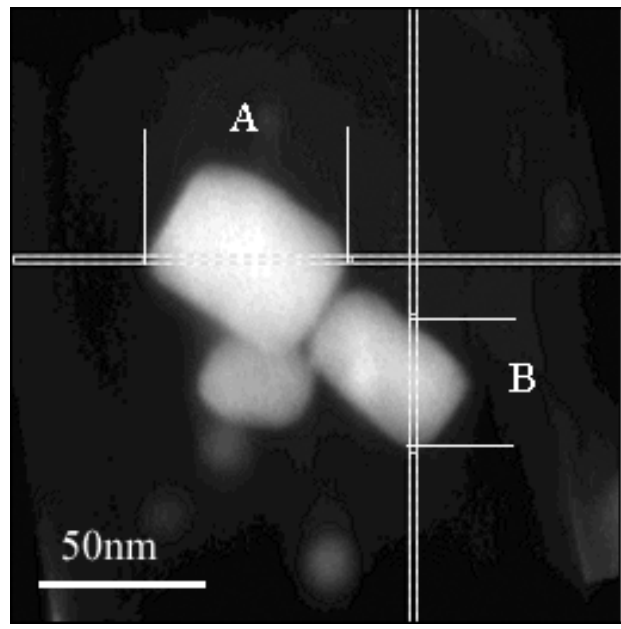

annular dark field (ADF) tilt-series

$300 \mathrm{~mm}$ camera length

range: -70 to $+70 ; 1$ degree increment acquistion time $32 \mathrm{~min}$

$512 \times 512$ pixel

$10 \mu$ s dwell time

density ratio: $\mathrm{A}^{*} \mathrm{~N}_{\mathrm{A}} /(\mathrm{B} * \mathrm{NB})=1.2$

FIG. 2 Projection along $\mathrm{z}$ axis of reconstructed volume from annular dark-field tilt-series

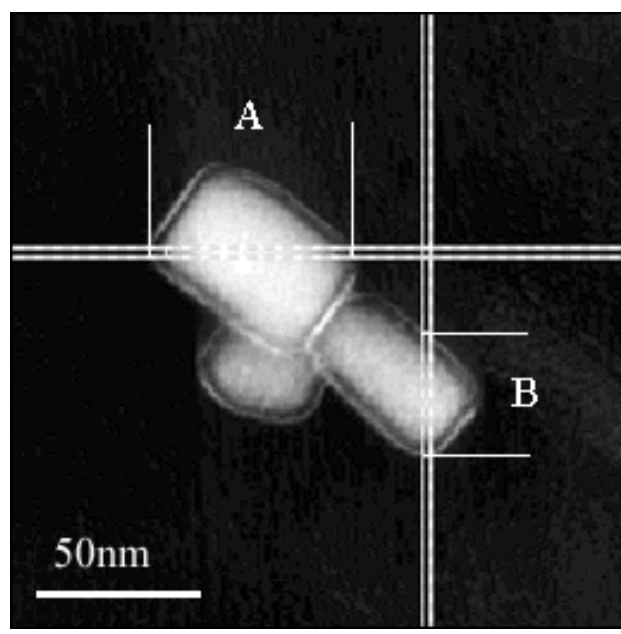

bright field tilt series

range: -70 to $+70 ; 1$ degree increment acquisition time $31 \mathrm{~min}$

$1 \mathrm{k} \times 1 \mathrm{k}$ CCD camera

1s exposure (rebinned by 2)

density ratio: $\mathrm{A}^{*} \mathrm{~N}_{\mathrm{A}} /(\mathrm{B} * \mathrm{NB})=1.1$

FIG. 3 Projection along $\mathrm{z}$ axis of reconstructed volume from bright-field tilt-series 